\title{
Nanodispersed $\mathrm{DO}_{3}$-phase nanostructures observed in magnetostrictive $\mathrm{Fe}-19 \%$ Ga Galfenol alloys
}

\author{
Somnath Bhattacharyya, ${ }^{1, *}$ J. R. Jinschek, ${ }^{1,2}$ A. Khachaturyan, ${ }^{3}$ H. Cao, ${ }^{1}$ J. F. Li, ${ }^{1}$ and D. Viehland ${ }^{1}$ \\ ${ }^{1}$ Department of Materials Science and Engineering Department, Virginia Tech, Blacksburg, Virginia 24061, USA \\ ${ }^{2}$ Institute for Critical Technology and Applied Science (ICTAS), Virginia Tech, Blacksburg, Virginia 24061, USA \\ ${ }^{3}$ Department of Materials Science and Engineering, Rutgers University, Piscataway, New Jersey 08854, USA
}

(Received 12 April 2007; revised manuscript received 27 November 2007; published 10 March 2008)

\begin{abstract}
Few nanometer large $(<2 \mathrm{~nm})$ inclusions of $\mathrm{DO}_{3}$-phase structure have been identified in the $A 2$ matrix of highly magnetostrictive $\mathrm{Fe}-19 \% \mathrm{Ga}$ alloys by high-resolution transmission electron microscopy (HRTEM). In addition, we have found that these nanostructures include a high density of $\{100\}$ line defects with a Burgers vector of $\frac{a_{b c c}}{2}\langle 100\rangle$. This dispersion of $\mathrm{DO}_{3}$-phase nanostructures formed within the main $A 2$ matrix and the lifting of their coherency by defects are consistent with a recent theory for the structure and properties of magnetostrictive Fe-Ga and Fe-Al alloys [A. G. Khachaturyan and D. Viehland, Metall. Mater. Trans. A 38A, 2308 (2007); 38A, 2317 (2007)].
\end{abstract}

DOI: 10.1103/PhysRevB.77.104107

PACS number(s): 87.64.Ee, 61.46.Df

\section{INTRODUCTION}

Giant magnetostriction in $\mathrm{Fe}-x \%$ Ga alloys $(15<x$ $<27$ at $\%$ ) offers potential for future generations of sensors and actuators. ${ }^{1} \mathrm{~A}$ maximum in the magnetostrictive strain of $3 / 2 \lambda_{100} \leqslant 400 \mathrm{ppm}$ is found at Ga content of about $19 \%$, which is ten times higher than that of pure $\alpha$-Fe. This alloy also offers a unique combination of other properties including high mechanical strength, good ductility, high-imposed blocking stress, and exceptional weakfield properties at moderate saturation fields. ${ }^{2,3}$ Accordingly, the alloy offers a large magnetoelastic energy density when used either in tension or compression.

Both equilibrium ${ }^{4}$ and metastable ${ }^{5}$ phase diagrams for $\mathrm{Fe}-x \% \mathrm{Ga}$ have been reported. In the equilibrium diagram, a disordered body-centered-cubic (bcc) $\alpha$-Fe (or A2) phase is in equilibrium with a face-centered-cubic (fcc) $L 1_{2}$ ordered one; whereas in the metastable diagram, the $A 2$ phase is in a metastable equilibrium with a bcc $\mathrm{DO}_{3}$ ordered phase. It is interesting that the metastable phase diagram of $\mathrm{Fe}-\mathrm{Ga}$ alloys is qualitatively and even quantitatively similar to the wellstudied phase diagram of the chemically similar Fe-Al system. This makes information about the Fe-Al system a helpful guide in studying the $\mathrm{Fe}-\mathrm{Ga}$ system. It has been established that the phase stability and magnetostrictive properties of $\mathrm{Fe}-\mathrm{Ga}$ are significantly dependent on the history of the sample, ${ }^{6}$ varying with annealing time, temperature, and cooling rate. This indicates that the enhanced magnetostriction is not an intrinsic property of the lattice, but rather might be due to an underlying inhomogeneity.

To explain the origin of enhanced magnetostriction in $\mathrm{Fe}-x \% \mathrm{Ga}$, Cullen et al. ${ }^{7}$ proposed that the bcc lattice of $\alpha$-Fe is strained along the [100] directions due to a directional short-range ordering of Ga atoms. For binary alloy additions to $\mathrm{Fe}$, the solutes form clusters that act as locally distorted anisotropic defects. These clusters can change their orientations under application of magnetic $(H)$ and/or stress $(\sigma)$ fields. Recently, an alternative mechanism for enhanced magnetostriction and elastic softening was proposed by Khachaturyan and Viehland. ${ }^{8,9}$ This mechanism is based on the assumption that the slow cooled bcc alloys are in fact in a structurally and chemically heterogeneous state consisting of a coarsening-resistant nanodispersion of a $\mathrm{DO}_{3}$ phase within an $A 2$ matrix, formed due to coherency lifting by excess vacancies. Coarsening resistance was a result of decomposition only being able to proceed in a two-phase field of the equilibrium stress-free diagram if the coherency between the phases responsible for misfit-generated stress was lifted. The only feasible coherency lifting mechanism operational starting from precipitate nucleation is vacancy absorption. Coarsening-resistant nanodispersion is known for the case of chemically similar Fe-Al and other alloys. ${ }^{10-14}$ It is sometimes referred to as the $K$ state. ${ }^{15,16}$

Khachaturyan and Viehland have assumed that this compositionally heterogeneous state leads to a cubic $(C)$ $\rightarrow$ tetragonal $(T)$ displacive transformation that brings the structure closer to an equilibrium fcc-based $L 1_{2}$ ordered phase. This also explains that giant extrinsic magnetostriction and elastic softening are then caused by the macroscopic strain generated by a magnetic field-induced displacive transformation or a stress-induced reorientation of tetragonal phase nanotwins. ${ }^{8,9}$

In this paper, $\mathrm{Fe}-19 \% \mathrm{Ga}$ has been examined on the nanometer scale using conventional transmission electron microscopy (TEM), high-resolution transmission electron microscopy (HRTEM), as well as HRTEM lattice image analysis and simulation. Our results demonstrate the presence of a $\mathrm{DO}_{3}$ nanodispersion within the $A 2$ matrix, where coherency is lifted by excess vacancies obtained by removing segments of the bcc lattice planes.

\section{EXPERIMENTAL PROCEDURE}

The $\mathrm{Fe}-19 \% \mathrm{Ga}$ alloy, used in present study, was grown at Ames Laboratory using the Bridgman method as described by Clark et al. ${ }^{17}$ This sample was further annealed for 3 days at $550{ }^{\circ} \mathrm{C}$ to reduce stress introduced during growth by rapid furnace cooling $\left(10^{\circ} \mathrm{C} / \mathrm{min}\right)$.

Single crystal specimens were cut in a direction perpendicular to the preferred electron beam direction [001] for 


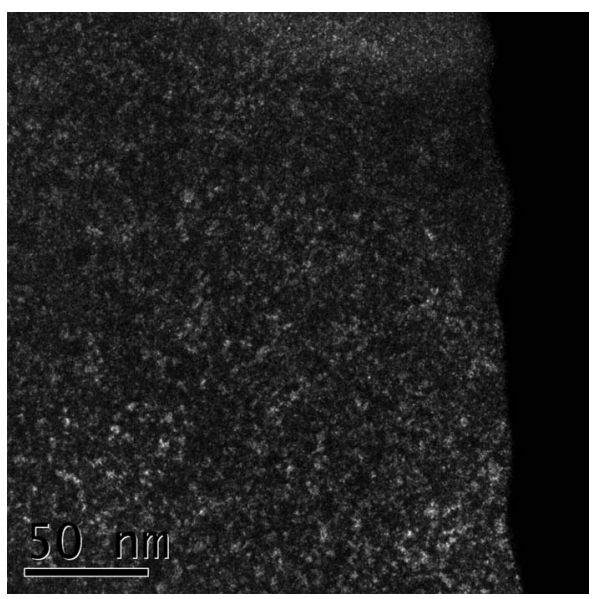

FIG. 1. Dark-field TEM image using (010) superlattice reflection of $\mathrm{DO}_{3}$ for $\mathrm{Fe}-19$ at $\% \mathrm{Ga}$.

transmission electron microscopy (TEM) observations. Specimens were prepared utilizing the standard techniques of grinding, dimpling, and argon ion-beam thinning (Model 1010 ion mill, E. A. Fischione Instrument, Inc., PA, USA). Ion-beam thinning was carried out on both sides of the sample at an inclination angle of $8^{\circ}$ of the ion beam with respect to the sample.

The HRTEM experiments were performed using an FEI Titan $^{\text {TM }}$ TEM, operated at $200 \mathrm{kV}$. This microscope was equipped with a Schottky field emission gun (FEG) and a postcolumn Gatan image filter (GIF) for energy filtering. Care was taken to orient the [001] zone axis of the specimen parallel to the incident electron beam before imaging. First, we performed energy dispersive analysis by $\mathrm{x}$-rays (EDAX) over numerous regions of several crystals. In all cases, we obtained a Ga content of $19 \pm 0.5$ at $\%$, clearly showing that the crystals was reasonably homogeneous.

Images were then captured onto a $1024 \times 1024$ pixel charge-coupled device (CCD) array. The acquisition time for high-resolution image was $4 \mathrm{~s}$. Digital micrograph software (Gatan, Inc., Pleasanton, CA, USA) was used to analyze these HRTEM lattice images. MacTempasX software (version 2.1.6) was used to perform HRTEM image simulation based on the multislice algorithm and by utilizing the determined microscope and sample parameters, as well as by subsequently comparing simulated and experimental HRTEM lattice images.

\section{RESULTS}

\section{A. Dark-field imaging: Demonstration of a nanostructure}

Figure 1 shows a dark-field image of the $\mathrm{Fe}-19 \% \mathrm{Ga}$ specimen in [001] zone-axis orientation taken using the (010) reflection. The image represents a very thin region of the sample close to hole in the TEM sample caused by ion milling to exclude dynamic scattering effects. The weak (010) reflection is the superlattice reflection of the $\mathrm{DO}_{3}$ structure present within the $A 2$ matrix. ${ }^{18}$ Another explanation for these $\{100\}$ superlattice reflections would be the presence

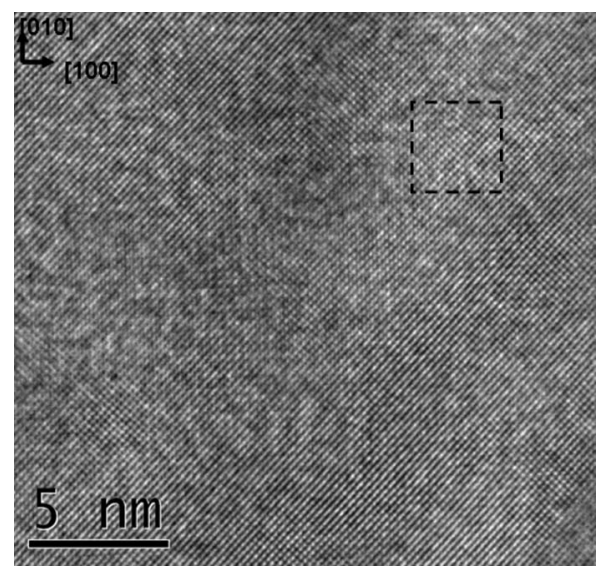

FIG. 2. High-resolution transmission electron micrograph (HRTEM). Region marked by the square (dotted line) indicates the region from which Fig. 4 (that will be shown below) was obtained by filtering.

of the $B 2$ phase. But the $B 2$ phase is only stable at high temperatures, ${ }^{4,5}$ and can thus be excluded.

The dark-field image in Fig. 1 clearly reveals a contrast consisting of a few nanometer wide bright regions within a dark matrix. This indicates the presence of $\mathrm{DO}_{3}$ nanostructures within the $A 2$ matrix. By analysis of the contrast, the size of these nanoregions is estimated to be between 2 and $8 \mathrm{~nm}$. The point-to-point resolution of the dark-field image depends on the size of the objective aperture that is used in selecting the diffraction spot of interest. The radius of the objective aperture in reciprocal space used in our experiment is $0.21 \AA^{-1}$. Therefore, the resolution of the dark-field image in Fig. 1 is $4.8 \AA$. Due to limited resolution the bright features in this figure are potentially mottled over various neighboring (smaller) regions. The Fe-19\% Ga alloy present in our sample is not a homogeneous bcc solution, but rather a nanodispersion of small clusters.

\section{B. Lattice and Fourier images: Demonstration that the nanostructure has the $\mathrm{DO}_{3}$ structure}

Figure 2 shows an image of this sample in high-resolution mode (HRTEM). The power spectrum, i.e., the square of the magnitude of the complex Fourier transformation (equal to the intensity distribution) of the HRTEM lattice image in Fig. 2 is shown in Fig. 3. This gives detailed information about the nanostructures revealed from the dark-field image.

The Fourier transform, as well as the power spectrum of the HRTEM image, indicate spatial frequencies (i.e., $g$ vectors) of lattice fringes that are present and interfere to form the lattice image in Fig. 2. This Fourier analysis of different spatial frequencies points to characteristic features in a HRTEM lattice image such as the known regular interference pattern, but in addition variations from the regular pattern caused by structural heterogeneity. In particular, the power spectrum in Fig. 3 reveals strong $\{110\}$ spots (indicated by solid circles) that correspond to fundamental lattice fringes common to both the disordered bcc and ordered $\mathrm{DO}_{3}$ structures, and weaker $\{010\}$ ones (indicated by dashed circles) 


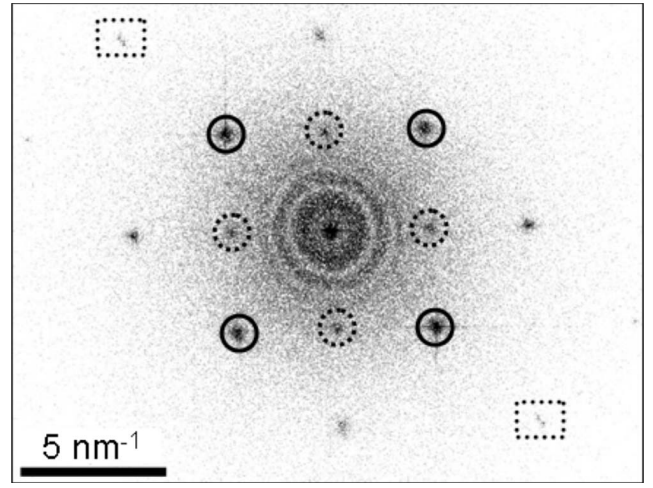

FIG. 3. Power spectrum of Fig. 2. Solid circles show $\{110\}$ reflections, dotted circles show $\{010\}$ reflections, and dotted lines show spot splitting at $\{220\}$ reflections.

that are believed to be $g$ values of superlattice fringes typical of the $\mathrm{DO}_{3}$ ordered structure. In the case of Fe-Ga, weak $\{010\}$ spots at room temperature are indicative of the $\mathrm{DO}_{3}$ phase. In addition, the power spectrum exhibited longitudinal splitting of the $\{220\}$ reflections (denoted by dotted squares in Fig. 3) along the (220) direction. Splitting along the (220) is consistent with the observation of cubic $\mathrm{DO}_{3}$ nanoprecipitates within the bcc matrix: a longitudinal splitting of all reflections is the result of the difference in lattice parameters between precipitate $\left(\mathrm{DO}_{3}\right)$ and parent $(A 2)$ phases under the condition that both phases are cubic and have different composition (the composition of the $\mathrm{DO}_{3}$ precipitates is expected to be close to that of the ideal $\mathrm{Fe}_{3} \mathrm{Ga}$ stoichiometry). In principle, splitting should also be present in the $\{110\}$ reflections closer to the central beam: however, it was not observed due to limited point-to-point resolution of the lattice image. Splitting is smaller for reflections closer to the central beam, and thus a split caused by two phase coexistence should be two times larger for (220) relative to (110) reflections.

To analyze the origin of the weak $\{010\}$ superlattice reflections in the Fourier transform of the HRTEM lattice image in Fig. 2, an inverse Fourier transformation operation (IFFT) was applied by taking only the $\langle 010\rangle g$ vectors into account. In the Fourier transform of the lattice image in Fig. 2 , the weak $\{010\}$ superlattice reflections of the $\mathrm{DO}_{3}$ phase were selected by smoothed edge masks of size $1.2 \mathrm{~nm}^{-1}$.

The inverse Fourier transformation of the masked Fourier transform results in an image that is an interference pattern containing only the $\langle 010\rangle$ spatial frequencies (from Fig. 2). Accordingly, this interference pattern contains only $\langle 010\rangle g$ vector contributions. Please note that weak $\{010\}$ reflection could potentially also be caused by dynamical scattering in the $A 2$ matrix. However, this possibility was excluded since the sample was sufficiently thin, as evidenced by the presence of Thon rings ${ }^{19}$ in Fig. 3, and by the fact that $\mathrm{DO}_{3}$ was present throughout the matrix. We can thus conclude that the inverse Fourier transformed image, which was formed including only the $\{010\}$ reflections, represents only the $\mathrm{DO}_{3}$ structure. Please note that it is only sharp features in the inverse Fourier transformed images which are of interest. This is because the blurred ones are an artifact produced by Fourier transformation of a masked region. A region of the

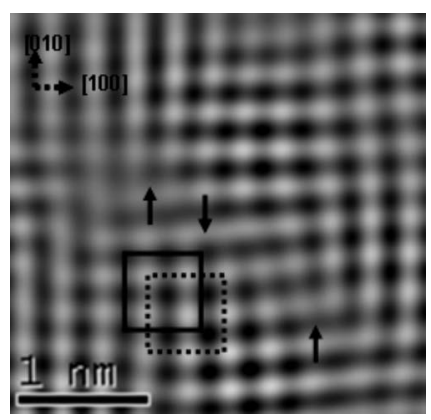

FIG. 4. Inverse Fourier transform of the $\{010\}$ reflections, which were obtained from the Fourier transform of the marked region in lattice image in Fig. 2. The image is an interference pattern that contains only the spatial frequencies (from Fig. 2) that are contained within smoothed edge masks of size $12 \mathrm{~nm}^{-1}$ placed about the weak $\{010\}$ superlattice reflections. Region $A$ and $B$ are surrounded by dotted and solid lines, respectively. Negative and positive extra half planes are indicated by arrows.

inverse Fourier transformed image that exhibited $\{010\}$ lattice fringes is shown in Fig. 4. This figure also indicates that the $\mathrm{DO}_{3}$ nanostructure contains many line defects.

Next, the $\{110\}$ reflections (defined by solid circles in the power spectrum in Fig. 3) in the Fourier transform of the HRTEM lattice image (Fig. 2) were taken into account. As described above, masking tools were applied on these spots. A region of the inverse Fourier transformed image formed only from the $\langle 110\rangle$ spatial frequencies in these masked areas is shown in Fig. 5. Although both $A 2$ and $\mathrm{DO}_{3}$ structures make contributions to these spots (spatial frequencies), the volume of the $\mathrm{DO}_{3}$ nanostructures inside the $A 2$ matrix is much smaller. So, it can be assumed that Fig. 5 is an image representative of the $A 2$ matrix phase. Please note there is no indication of line defects in the matrix phase in contrast to what we observed in the $\mathrm{DO}_{3}$ phase in Fig. 4 .

The contrast in the IFFT image in Fig. 4 depicts the $\mathrm{DO}_{3}$ nanostructure. It is important to point out that numerous edge

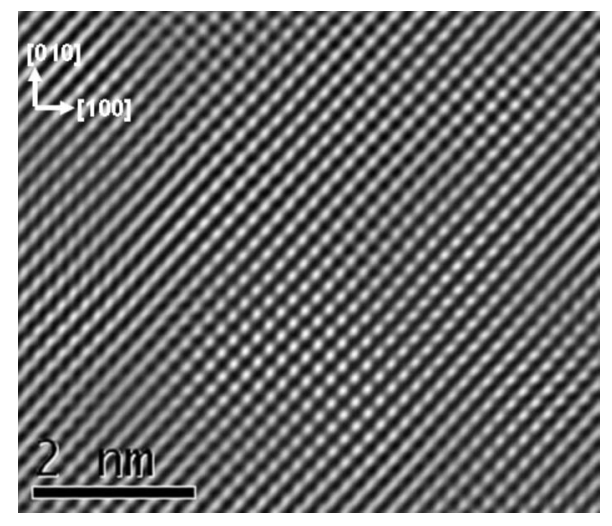

FIG. 5. Inverse Fourier transform of the $\{110\}$ reflections, which were obtained from the Fourier transform of the lattice image in Fig. 2. The image is an interference pattern that contains only the spatial frequencies (from Fig. 2) that are contained within smoothed edge masks of size $12 \mathrm{~nm}^{-1}$ placed about the strong $\{110\}$ reflections. 

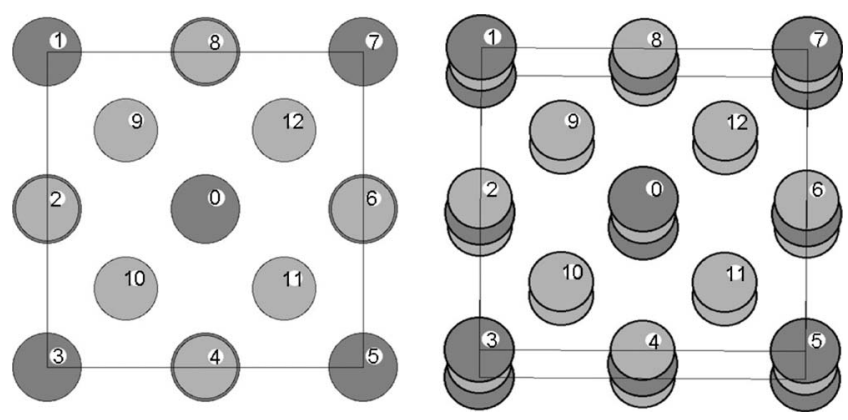

FIG. 6. (a) Crystal structure of $\mathrm{DO}_{3}$ where dark and light colored circles represent $\mathrm{Ga}$ and Fe atoms, respectively. (b) Projection of this crystal structure along the [001] direction. Columns are numbered from 0 to 12 in both images to show the positions of the projections of each column.

line defects are present inside these nanostructures: both negative and positive extra half planes that originate inside the $\mathrm{DO}_{3}$ phase are indicated by arrows in Fig. 4. The Miller indices of these extra half planes need to be determined to identify the features that are responsible for their appearance.

\section{Simulated lattice images}

HRTEM lattice image simulation by multislice algorithm was performed using the crystal structure of $\mathrm{DO}_{3}$ in $\mathrm{Fe}-\mathrm{Ga}$ alloys, shown in Fig. 6(a). The projection of this structure along the [001] direction is given in Fig. 6(b). The lattice parameter of $\mathrm{DO}_{3}$ was assumed to be $5.799 \AA$, following Lograsso et al. ${ }^{6}$ A virtual objective aperture was used in the simulation to allow only the weak $\{010\}$ spots to contribute to image formation.

Since $A 2$ and $\mathrm{DO}_{3}$ have cubic structures, their structural unit size could be treated as uniform in all $\langle 100\rangle$ directions. Therefore, it can be assumed that the dimension (thickness) of the $\mathrm{DO}_{3}$ nanophase inclusion along the electron beam ([001]) is the same as in the other two directions ([100] and [010]) present in the image (length and width of about 4 unit cells each). Experimental parameters were used as simulation parameters. For example, the positions of the circular rings (Thon rings) surrounding the central spot in the power spectrum (Fig. 3) were used to determine the defocus of the area imaged in Fig. 2. By matching the ratio of the radius of the first and second Thon rings with the ratio of the first and second zero crossings of the particular phase contrast transfer function $(\mathrm{CTF})$ of our FEI Titan microscope, the average defocus of the imaged area has been determined to be $-131( \pm 2) \mathrm{nm}$ (where the sign "-" denotes underfocus).

Two regions of inverse contrast $(A$ and $B$ ) were selected in the image presented in Fig. 4. Rapid oscillation of the CTF of a FEG TEM cause contrast inversion in the lattice image, when the defocus and/or thickness are changed. A thicknessdefocus map of HRTEM lattice images of the $\mathrm{DO}_{3}$ structure was simulated for comparison. By calculation and comparison of the $\chi^{2}$ difference between simulated and experimental images, the positions of the atomic columns in the $\mathrm{DO}_{3}$ phase, and thereby the position of the extra half planes, were determined. The "best fit" (least $\chi^{2}$ ) simulated images for
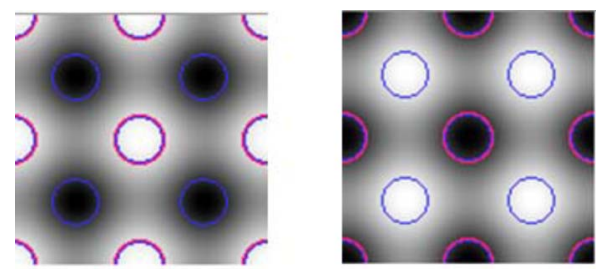

FIG. 7. (Color online) Simulated images (with calibration 1 pixel $=0.02 \mathrm{~nm}$ ) with atom overlay. Blue and red circles represent Fe and Ga atoms, respectively, (a) at thickness of $4 \pm 1$ unit cell and defocus of $-134 \mathrm{~nm}$ and (b) at thickness of $1.5 \pm 0.5$ unit cell and defocus of $-120 \mathrm{~nm}$.

both of the regions are shown in Figs. 7(a) and 7(b), respectively, including the overlay of the atomic columns. The best $\chi^{2}$ fit values between simulation and experiment for both regions $A$ and $B$ were comparable to each other. However, only the defocus value $(-134 \mathrm{~nm})$ and the thickness $(4 \pm 1$ unit cells) of the simulated image presented in Fig. 7(a) (that matches with region $A$ in Fig. 4) agreed well with the determined average defocus of the entire image area and with the dimensions (length and width) of the nanostructure in Fig. 4. Thus, it can be concluded that the black features in the IFFT image in Fig. 4 are the projections of the atomic columns containing only $\mathrm{Fe}$ atoms, and that the white features are of the columns containing both Fe and Ga atoms [see Fig. 7(a)].

Comparison of the atomic projection shown in Fig. 7(a) with the structural model in Fig. 6(b) revealed that the extra half planes are $\{400\}$ planes. Due to a high line defect density in Fig. 4, the determination of the Burgers vector is very complicated. Under the assumption that all extra half planes in the $\mathrm{DO}_{3}$ nanostructures in the imaged area are $\{400\}$ planes, the Burgers vector can be determined from another $\mathrm{DO}_{3}$ nanostructure, as illustrated in Fig. 8. The in-plane projection of the Burgers vector is $\frac{a}{2}\langle 100\rangle$, where $a$ is the lattice parameter of $\mathrm{DO}_{3}$. This type of $\langle 100\rangle$ line defects was previously reported in the $\mathrm{DO}_{3}$ structure of the $\mathrm{Fe}-\mathrm{Al}$ system by Munroe and Baker. ${ }^{20}$

\section{DISCUSSION}

Our investigations reveal that $\mathrm{Fe}-19 \% \mathrm{Ga}$ alloys are in fact a structurally and chemically heterogeneous state consisting of a dispersion of nanosized precipitates within an $A 2$

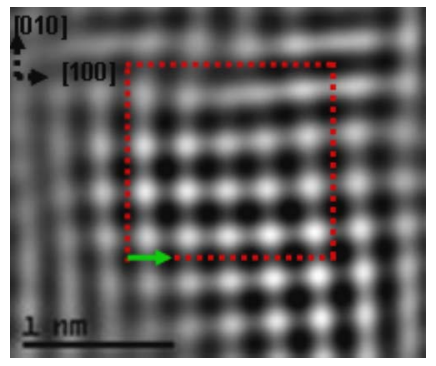

FIG. 8. (Color online) Determination of the Burgers vector of negative half planes in the $\mathrm{DO}_{3}$ nanostructure. Dotted box shows the Burgers circuit and the arrow shows the Burgers vector. 
matrix phase. Comparisons of experimental and simulated HRTEM lattice images confirm that the nanodispersed precipitates are of $\mathrm{DO}_{3}$ phase. ${ }^{5}$ Fourier analysis of HRTEM lattice images showed that the $\mathrm{DO}_{3}$ precipitates consisted of fragments $4-6$ unit cells in size (oriented along $\langle 001\rangle$ directions). These findings are consistent with on-going synchrotron investigations by McQueeny of ordering in Fe-Ga alloys, ${ }^{21}$ which have revealed extremely diffuse (100) superlattice reflections for the $\mathrm{DO}_{3}$ phase with an estimated correlation length for the ordered structure of about 5 unit cells. Similar types of $\mathrm{DO}_{3}$ precipitates have been previously observed in the chemically similar $\mathrm{Fe}-\mathrm{Al}$ alloys within the solubility limit of the $A 2$ phase. $^{10,11}$ Together, our HRTEM data in real space and McQueeny's structural results in reciprocal space provide strong evidence of the existence of a nanosized structurally heterogeneous $\mathrm{DO}_{3}$ phase dispersed within an $A 2$ matrix-as recently proposed theoretically by Khachaturyan and Viehland. ${ }^{8,9}$

Ikeda et $a l^{5}$ previously reported the observation of small $\mathrm{DO}_{3}$ regions within an $A 2$ matrix for $\mathrm{Fe}-19 \% \mathrm{Ga}$ and $\mathrm{Fe}-20 \% \mathrm{Ga}$, which were about $40-80 \mathrm{~nm}$ in size. For $\mathrm{Fe}-22 \% \mathrm{Ga}$, these $\mathrm{DO}_{3}$ regions coarsened to sizes of $>400 \mathrm{~nm}$ upon thermal annealing at temperatures slightly below the $\mathrm{A} 2-\mathrm{DO}_{3}$ boundary. The size of our nanoregions for $x=19$ at $\% \mathrm{Ga}$ are smaller than those of Ikeda et al. $;{ }^{5}$ however, they are of similar size as those previously reported in Fe-Al alloys in the $K$ field, ${ }^{17,18}$ and to that of McQueeny et al. $^{21}$ by synchrotron radiation for $\mathrm{Fe}-19 \% \mathrm{Ga}$. Furthermore, ongoing investigations by our research team have shown similar coarsening-resistant nanodispersions of $\mathrm{DO}_{3}$ in an $A_{2}$ matrix for $10<x<20$ at $\%$, which will be published at a later date after more thorough studies are completed. This is consistent with prior investigations of coarseningresistant $\mathrm{DO}_{3}$ nanodispersion in an $A 2$ matrix within the $K$ field of $\mathrm{Fe}-x \% \mathrm{Al}$ for $6<x<19$ at $\% \mathrm{Al}^{22,23,10,11}$ The larger size of the $\mathrm{DO}_{3}$ particles reported by Ikeda et al. ${ }^{5}$ are for compositions not in the $K$ field (i.e., for $x>\rho>22$ at $\%$ ), but rather in the two phase field of the coherent phase diagram where conventional nucleation and growth can occur, as again shown for $\mathrm{Fe}-x \% \mathrm{Al}$ for $x>19$ at $\% \mathrm{Al}^{22,23,10,11}$

According to Khachaturyan and Viehland, ${ }^{8,9}$ a coarseningresistant dispersion of $\mathrm{DO}_{3}$ nanoparticles is formed within the composition range adjacent to the $A 2$ phase solubility limit. Its formation is made possible by the lifting of coherency between $\mathrm{DO}_{3}$ precipitate and $A 2$ phases, which should occur by absorption of excess vacancies that removes excess volume caused by formation of Ga-enriched $\mathrm{DO}_{3}$ particles. In fact, we also observed a high concentration of defects within $\mathrm{DO}_{3}$-phase nanoprecipitates, in contrast to the defectfree $A 2$ matrix. Lattice imaging of the $\mathrm{DO}_{3}$ regions revealed the line defects along (100) planes (i.e., the presence of additional $\{400\}$ planes, with a Burgers vector of $\left.\frac{a}{2}\langle 100\rangle\right)$.

Although the determination of the origin of these extra planes is still part of ongoing research, prior investigations of Fe-Al alloys indicate excess vacancies and line defects possessing such Burgers vector. ${ }^{20}$ Our findings may provide insights into specific structural details of vacancy condensation, in particular when compared to recent diffuse neutron investigations by Cao et al. ${ }^{24}$ which have shown diffuse rods along $\langle 100\rangle$. This comparison suggests that the $\{100\}$ line defects may actually be vacancy loops along $\{100\}$ planes. Formation of such vacancy loops would be consistent with the structurally heterogeneous model for $\mathrm{Fe}-\mathrm{Ga}$ alloy, ${ }^{8,9}$ where the $\mathrm{DO}_{3}$ nanoparticles accommodate the elastic strain by removing (100) extra planes of the $\mathrm{DO}_{3}$ precipitates and thus becoming noncoherent.

The current model of the nanodispersion mechanism ${ }^{8,9}$ is based on the thermodynamic theory of a vacancy-assisted nanodispersion of the ordered phase in the single-phase field of the coherent phase diagram, except in the two-phase field of the equilibrium (stress-free) phase diagram previously developed. ${ }^{22,23}$ According to Khachaturyan and Viehland, ${ }^{8,9}$ the mechanism of nanodispersion formation is the following. Even though both coexisting phases $\left(\mathrm{A}_{2}\right.$ and $\left.\mathrm{DO}_{3}\right)$ are cubic, the volume of the Ga-enriched $\mathrm{DO}_{3}$ precipitates $\left(\mathrm{Fe}_{3} \mathrm{Ga}\right)$ is larger than that of the $A 2$ matrix from which it decomposed. This is a direct consequence of the fact that the atomic radius of $\mathrm{Ga}$ is greater than that of $\mathrm{Fe}$. The Ga-enrichment-induced extra volume produced by the crystal lattice expansion generates elastic strain suppressing the $\mathrm{DO}_{3}$-phase precipitation in the coherent state. Our current understanding is that a "condensation" of vacancies should remove this extra volume of the precipitate phase, eliminating the source of elastic strain and lifting the coherency of the precipitates. This would result in the elimination of the corresponding elastic energy, making thermodynamically the precipitation of incoherent $\mathrm{DO}_{3}$ particles feasible.

Following this model, the size $D$ of the $\mathrm{DO}_{3}$ precipitates can be estimated from the following consideration. We assumed that the $\mathrm{DO}_{3}$ nanoparticles expand relative to the matrix by

$$
D \frac{a_{\mathrm{DO}_{3}} / 2-a_{A 2}}{a_{A 2}} .
$$

The size change $\Delta D$ should be close to (but less than) the amount $a_{A 2} / 2$ that has to be removed by an excess vacancy to reduce the transformation hindering stress. This leads to the following inequality:

$$
D<\frac{a_{A 2}^{2}}{2\left(a_{\mathrm{DO}_{3}} / 2-a_{A 2}\right)} .
$$

According to this inequality, a precipitate can form even when the excess volume of the precipitates is not completely eliminated by the volume of excess vacancies, but rather before this condition is achieved. Considering the values for the lattice parameters of the $A 2\left(a_{A 2} \approx 2.87 \AA\right)$ and $\mathrm{DO}_{3}$ with an ideal $\mathrm{Fe}_{3} \mathrm{Ga}$ stoichiometry $\left(a_{\mathrm{DO}_{3}} / 2 \approx 2.91 \AA\right)$ given by Massalski, ${ }^{25}$ the estimated size of the $\mathrm{DO}_{3}$ precipitates from Eq. (1) is $D<10 \mathrm{~nm}$. This value is consistent with our size observation of the $\mathrm{DO}_{3}$ nanoparticles by conventional TEM and HRTEM.

\section{CONCLUSIONS}

$\mathrm{Fe}_{3} \mathrm{Ga} \mathrm{DO}_{3}$-phase structures with a size of $\sim 2 \mathrm{~nm}$ have been investigated in highly magnetostrictive $\mathrm{Fe}-19 \% \mathrm{Ga}$ Galfenol alloys using conventional TEM as well as HRTEM 
imaging and simulation. In agreement with the previous prediction of Khachaturyan and Viehland ${ }^{8,9}$ and the Fe-Ga phase diagram, it has been shown that this $\mathrm{Fe}-19 \%$ Ga solid solution in fact consists of a nanodispersion of $\mathrm{DO}_{3}$ phase precipitates in the $A 2$ matrix. The presence of $\{400\}$ line defects in the $\mathrm{DO}_{3}$ nanoprecipitates was found by reference to a uniform $A 2$ matrix. The presence of $\{400\}$ line defects inside the nanoprecipitates can be explained by the appearance of vacancy loops to compensate the $\mathrm{Ga}$ enrichment in $\mathrm{Fe}_{3} \mathrm{Ga}$ of $\mathrm{DO}_{3}(25 \% \mathrm{Ga})$ in comparison to the nominal $19 \%$ Ga content of the solid Galfenol solution. The appearance of vacancy loops is very likely since this would lift the coherency of the $\mathrm{DO}_{3}$ phase precipitates, reduce stress, and provide the thermodynamic conditions required for the $\mathrm{DO}_{3}$ phase precipitation within this $\mathrm{Fe}-\mathrm{Ga}$ composition range.

\section{ACKNOWLEDGMENTS}

This work was supported by the Office of Naval Research through MURI Contracts No. N00014-06-1-0530 and No. N00014-06-1-0204. The authors acknowledge support of the staff and facilities at the Nanoscale Characterization and Fabrication Laboratory (NCFL) at ICTAS, Virginia Tech.

*somnath.tem@gmail.com

${ }^{1}$ Handbook of Giant Magnetostrictive Materials, edited by G. Engdhal (Academic, San Diego, 2000).

${ }^{2}$ S. Guruswamy, N. Srisukhumbowornchai, A. E. Clark, J. B. Restorff, and M. Wun-Fogle, Scr. Mater. 43, 239 (2000).

${ }^{3}$ A. E. Clark, J. B. Restorff, M. Wun-Fogle, T. A. Lograsso, and D. L. Schlagel, IEEE Trans. Magn. 36, 3238 (2000).

${ }^{4}$ H. Okamoto, in Phase Diagrams of Binary Iron Alloys, edited by H. Okamoto (ASM International, Materials Park, OH, 1993), p. 147.

${ }^{5}$ O. Ikeda, R. Kainuma, I. Ohnuma, K. Fukamichi, and K. Ishida, J. Alloys Compd. 347, 198 (2002).

${ }^{6}$ T. A. Lograsso, A. R. Ross, D. L. Schlagel, A. E. Clark, and M. Wun-Fogle, J. Alloys Compd. 350, 95 (2003).

${ }^{7}$ J. R. Cullen, A. E. Clark, M. Wun-Fogle, J. B. Restorff, and T. A. Lograsso, J. Magn. Magn. Mater. 226, 948 (2001).

${ }^{8}$ A. G. Khachaturyan and D. Viehland, Nucl. Metall. 38A, 2308 (2007)

${ }^{9}$ A. G. Khachaturyan and D. Viehland, Nucl. Metall. 38A, 2317 (2007).

${ }^{10}$ H. Warlimont and G. Thomas, Met. Sci. J. 4, 47 (1970).

${ }^{11}$ D. Watanabe, H. Morita, H. Saito, and S. Ogawa, J. Phys. Soc. Jpn. 29, 722 (1970).

${ }^{12}$ W. Gaudig and H. Warlimont, Z. Metallkd. 60, 488 (1969).

${ }^{13}$ J. H. Spruel and R. R. Stanabury, J. Phys. Chem. Solids 26, 811 (1965).

${ }^{14}$ E. Ruedl, P. Delavign, and S. Amelinck, Phys. Status Solidi 28, 305 (1968).

${ }^{15}$ H. Thomas, Z. Metallkd. 41, 185 (1950).

${ }^{16}$ H. Thomas, Z. Phys. 129, 219 (1951).

${ }^{17}$ A. E. Clark, M. Wun-Fogle, J. B. Restorff, and T. Lograsso, in Proceedings of the Fourth Pacific Rim International Conference on Advanced Materials and Processing (PRICM4), edited by S. Hanada, Z. Zhong, S. Nam, and R. Wright (The Japan Institute of Metals, Sendai, 1996), p. 1711.

${ }^{18}$ L. Libao, F. Shiyou, L. Goudong, W. Guangheng, S. Xiudong, and L. Jianqi, Physica B 365, 102 (2005).

${ }^{19}$ F. Thon, Z. Naturforsch. A 21 (4), 476 (1966).

${ }^{20}$ P. R. Munroe and I. Baker, J. Mater. Sci. 28, 2299 (1993).

${ }^{21} \mathrm{R}$. McQueeney (private communication).

${ }^{22}$ A. G. Khachaturyan, Fiz. Tverd. Tela (S.-Peterburg) 13, 2417 (1971).

${ }^{23}$ A. G. Khachaturyan, The Theory of Structural Transformations in Solids (Wiley, New York, 1983).

${ }^{24}$ H. Cao, J. F. Li, D. Viehland, Z. Xu, and P. M. Gehring (unpublished).

${ }^{25}$ Binary Alloy Phase Diagram, edited by T. B. Massalski, 2nd ed. (ASM International, Materials Park, OH, 1990), p. 1064. 